\title{
Gestión curricular y desarrollo de la competencia digital docente en la formación inicial del profesorado
}

\section{Curriculum management and development of teaching digital competence in initial teacher training}

\author{
Alba Ruth Pinto-Santos \\ Universidad de La Guajira. Maicao, Colombia \\ arpinto@uniguajira.edu.co \\ Adolfina Pérez Garcias \\ Universidad de Las Islas Baleares. Palma, España \\ fina.perez@uib.es
}

\begin{abstract}
Resumen
El presente artículo analiza la incidencia que tiene la gestión curricular de la Formación Inicial Docente (FID) en el desarrollo de la Competencia Digital Docente (CDD) de los futuros profesores. Para tal efecto, a nivel metodológico se implementó un enfoque de investigación evaluativa. La muestra fue conformada por los estudiantes (n: 153) del programa licenciatura en educación infantil de la Universidad de La Guajira. Los instrumentos de recolección de datos usados fueron el inventario de indicadores de la gestión curricular, y una rúbrica para evaluar la competencia digital docente. Los resultados evidencian que existen a nivel curricular, objetivos y asignaturas que orientan la formación pedagógica en el uso educativo de la tecnología digital. No obstante, en la valoración de la CDD en los estudiantes se ubicaron en los niveles más bajos de la rúbrica, con 37,3\% en el nivel Inferior, y $30,7 \%$ en el nivel de Bajo. Se concluye que no se refleja tendencias de innovación educativa y procesos integradores de la CDD, y que existe la necesidad de fortalecer los procesos de formación en la FID para que los futuros docentes desarrollen las competencias que le permita una utilización eficaz de la tecnología digital a nivel profesional.
\end{abstract}

Palabras clave: Competencia Digital Docente (CDD), Formación Inicial Docente (FID), Investigación Evaluativa, Gestión Curricular, Educación Infantil.

\begin{abstract}
This paper analyzes the impact of the curricular management of Initial Teacher Training (ITT) curriculum has on the development of the Teaching Digital Competence (TDC) of future teachers. For this purpose, at the methodological level, an evaluative research approach was implemented. The sample was made up of students (n: 153) from the bachelor of early childhood education program at the University of La Guajira. The data collection instruments used were an observation sheet of indicators of curricular management, and a rubric to evaluate the teaching digital competence. The results show that there are at the curricular level, objectives and subjects that guide pedagogical training in the educational use of digital technology. However, in the assessment of TDC in students, they are located at the lowest levels of the rubric, with $37.3 \%$ at the Lower level, and $30.7 \%$ at the Low level. It is concluded that trends in educational innovation and integrative processes of the TDC are not reflected, and that there is a need to strengthen the training processes in the ITT so that future teachers develop the competencies that allow an effective use of digital technology to professional level.
\end{abstract}

RED. Revista de Educación a Distancia. Núm. 69, Vol. 22. Artíc. 8, 30-Ene-2022

DOI: http://dx.doi.org/10.6018/red.493551 
Key words: Teaching Digital Competence (TDC), Initial Teacher Training (ITT), Evaluative Research, Curricular Management, Early Childhood Education.

\section{Introducción}

La pandemia ha generado cuestionamientos sobre si los futuros docentes están preparados para afrontar los desafíos de la educación actual, donde existe alta presencia de la tecnología digital (Pozas \& Letzel, 2021). Esto teniendo presente que, aunque los docentes en formación usan ampliamente la tecnología, carecen de conocimientos pedagógicos para integrarlos de manera efectiva (Luik, Taimalu \& Suviste, 2018; Roulston, Cowan, Brown, Austin \& O’Hara, 2019). Asimismo, los escenarios emergentes de aprendizaje y los espacios laborales demandan del docente amplios conocimientos, habilidades y actitudes sobre el uso de la tecnología digital, competencias informacionales, uso seguro, reflexivo y crítico de los diferentes recursos digitales, trabajo en equipo, resolución de problemas, entre otros (Roll y Ifenthaler, 2021), lo cual representa nuevos roles y genera retos en procesos de formación e innovación educativa.

Así, la mirada se centra en los programas de formación del profesorado, teniendo presente que el desarrollo profesional del docente parte de la formación inicial centrada en competencias pedagógica, de gestión, de uso de herramientas y recursos digitales, y conocimientos de las asignaturas (Unesco, 2019). En este sentido, la formación inicial docente (FID) debe permitirle al futuro docente incorporar, modelar y desarrollar las competencias para diseñar entornos de aprendizaje y aprovechar la tecnología digital para mejorar los aprendizajes de los estudiantes (Borthwick \& Hansen, 2017; Graziano, Herring, Carpenter, Smaldino \& Finsness, 2017; Masoumi, 2021). Asimismo, en la FID se debe avanzar en los diferentes niveles y dimensiones de desarrollo de la Competencia Digital Docente (CDD) (Unesco, 2019; INTEF, 2017; Cabero-Almenara y Martínez, 2019; Pinto, Pérez, Darder, 2020).

La CDD es entendida como multidimensional (Ramírez-Montoya, Mena, RodríguezArroyo, 2017), se relaciona con el desarrollo de capacidades y actitudes para aprovechar la tecnología digital el ejercicio profesional docente (Castañeda, Esteve \& Adell, 2018; Esteve, Castañeda \& Adell, 2018). En este sentido, la CDD se asocia a la apropiación pedagógica de las TIC (Flores, 2014), a las capacidades de adaptación y transformación de las metodologías de enseñanza (Silva et al., 2018), a los procesos de innovación educativa con el uso de la tecnología digital (Amaya, Zúñiga, Salazar \& Ávila, 2018), y a la formación permanente del profesorado (Alonso-Ferreiro, 2018; Tejada \& Pozos, 2018).

No obstante, la apropiación de las TIC en los procesos pedagógicos tiene poca presencia o no se analiza con profundidad en los currículos de la FID (Tiede, 2020). Se considera que hace falta planes integrales para la preparación del futuro docente frente a la apropiación de la tecnología digital en las prácticas pedagógicas (Buss, Wetzel, Foulger \& Lindsey, 2015; Masoumi, 2021), ya que existen pocas oportunidades de desarrollar conocimientos avanzados relacionados con la tecnología educativa, por el poco el tiempo dedicado a este tipo de tareas en la FID (Voogt \& McKenney, 2017), y por otra parte, los planes de estudio de la formación del profesorado no garantizan un proceso de aprendizaje a lo largo de la vida y el desarrollo de la CDD (Pozas \& Letzel, 2021).

Gestión curricular y desarrollo de la competencia digital docente en la formación inicial del profesorado. Alba Ruth Pinto Santos y Adolfina Pérez Garcias.

Página 2 de 21 
A partir de los planteamientos anteriores, se reconoce la importancia que tienen los procesos de innovación y apropiación de las TIC tanto para el desarrollo de la CDD, como para el mejoramiento continuo de los estándares curriculares. En este orden de ideas, cabe resaltar que la gestión curricular en la FID constituye un conjunto de procesos correspondientes al diseño, desarrollo, focalización, articulación y evaluación del currículo escrito, enseñado y eficientemente aprendido, que promueve la formación de profesionales altamente capacitados y con las competencias docentes para generar transformaciones pedagógicas (Rohlehr, 2006). Adicionalmente, surge la necesidad de analizar el macroproceso de la gestión curricular asociado con la definición de metas internas y requerimientos externos, a partir de la planificación de estrategias de aprendizaje y las competencias a desarrollar en los estudiantes de acuerdo con el contexto académico, social, y profesional del proceso educativo (López, 2010).

En este sentido, es importante que desde la FID los docentes sean competentes en el uso efectivo de las TIC en los procesos pedagógicos, y desde la gestión curricular de los programas de formación del profesorado se logre atender las actitudes y la autoeficiencia de los futuros docentes y promover experiencias de aprendizaje genuinas y vinculadas a las prácticas pedagógicas que promuevan la CDD (Pozas \& Letzel, 2021). Las instituciones formadoras de docentes deben ser gestoras del conocimiento pedagógico, y deben ir más allá de promover los conocimientos disciplinares a un análisis y comprensión de la escuela, de acuerdo a las necesidades y retos de cada contexto, y el avance de las TIC (Ministerio de Educación de Colombia, 2013). Asimismo, se deben propiciar oportunidades para que el futuro docente observe, reflexione, y ponga en práctica en los contextos escolares la CDD (Guo, Ellis, Hong, 2020).

Por consiguiente, es fundamental la colaboración entre escuelas, universidades y líderes de asociaciones profesionales para promover el desarrollo de la CDD desde la FID (Borthwick \& Hansen, 2017), direccionar y rediseñar los programas de la FID atendiendo a las necesidades profesionales actuales (Graziano et al., 2017; Vaillant, 2019), y evaluar la efectividad de los programas de formación en integración de la tecnología educativa (Kaplon-Schilis \& Lyublinskaya, 2020). Es necesario en el desarrollo de las capacidades para apropiar la tecnología digital mayor responsabilidad política, inversión en formación docente, acciones en la FID, establecer metas nacionales e institucionales, y mantener un compromiso con el desarrollo profesional y parmente de los docentes (Unesco, 2019).

En Colombia se considera que faltan mayores esfuerzos en la FID para la apropiación de la tecnología digital en los procesos pedagógicos, ya que hay una desconexión a nivel curricular entre la formación del futuro docente y el escenario de la practica educativa (Ministerio de Educación de Colombia, 2013), y los programas formadores de maestros deben pasar de propuestas frente a la formación en el uso instrumental de la tecnología digital a apuestas holísticas de apropiación crítica, reflexiva y situada de la tecnología educativa en la profesionalización docente (Pinto et al., 2021). A partir de los referentes y planteamientos anteriormente expuestos, se plantea la siguiente pregunta central que orienta el presente estudio ¿Cuál es la incidencia de los aspectos curriculares de la formación inicial docente en los niveles de desarrollo de la competencia digital docente de los futuros profesores de educación infantil?.

Gestión curricular y desarrollo de la competencia digital docente en la formación inicial del profesorado. Alba Ruth Pinto Santos y Adolfina Pérez Garcias.

Página 3 de 21 
En este contexto, surge un escenario de investigación curricular aplicado a la FID con la implementación de los procesos de innovación pedagógica mediada por las TIC, desde una perspectiva articulada con las competencias profesionales de los futuros docentes que actualmente se caracteriza por el énfasis en el desarrollo de la CDD para responder de forma efectiva a las necesidades y retos educativos contemporáneos y por lo tanto se considera pertinente avanzar en la gestión curricular bajo los lineamientos y estándares esperados del aseguramiento de la calidad educativa. En consecuencia, el objetivo general contemplado para el desarrollo del presente estudio, consiste en analizar la incidencia que tiene la gestión curricular de la Formación Inicial Docente (FID) en el desarrollo de la Competencia Digital Docente (CDD) de los futuros profesores.

\section{Método}

El abordaje metodológico del presente estudio asume un enfoque de investigación evaluativa (López, 2014; Escudero, 2016), centrado en la verificación de las contribuciones del currículo al desarrollo de la CDD en la FID. La investigación evaluativa permite realizar juicios fundamentados sobre el fenómeno de estudio desde una mirada reflexiva y crítica, posibilitando la gestión, planificación y toma de decisiones para ofrecer soluciones a problemas concretos (Escudero, 2016). Asimismo, pretende usar el conocimiento teórico en la solución de problemas prácticos, desde el uso de metodologías de investigación flexibles (Escudero, 2009; 2016).

\section{Muestra}

La población objetivo correspondió a los estudiantes del Programa de Licenciatura en Educación Infantil de la Universidad de La Guajira y la muestra fue conformada utilizando como criterios de inclusión, en primera instancia la selección de estudiantes que actualmente han cursado los niveles superiores (VII a X semestre) equivalentes a los dos últimos años de formación en el pregrado, y en segunda instancia la condición de estar adelantando prácticas pedagógicas.

La muestra fue conformada con la participación de (n:153) estudiantes, los cuales pertenecen a los semestres VII (16,3\%), VIII (34\%), IX (30,7\%) y X (19\%). Sus edades fueron clasificadas en cinco rangos entre los 18 y 41 años, siendo el rango comprendido entre los (20 y 24) años el más representativo con un 61,4\% de la muestra total. Cabe resaltar que, en este caso frente a la caracterización por género, la muestra de estudiantes en su totalidad fue conformada por las mujeres.

\section{Instrumentos}

El primer instrumento fue el inventario de observación de indicadores de la gestión curricular de la CDD en la FID que estuvo conformado por 10 criterios de evaluación agrupados en 3 dimensiones relacionadas con la Integralidad, Flexibilidad, e Interdisciplinariedad de la CDD en el currículo. Este instrumento fue elaborado, basado en la propuesta de diagnóstico de factores académicos de la gestión curricular por competencias (López, 2010) y validado por 5 expertos en el ámbito de la tecnología educativa.

Gestión curricular y desarrollo de la competencia digital docente en la formación inicial del profesorado. Alba Ruth Pinto Santos y Adolfina Pérez Garcias. 
En términos conceptuales, cabe resaltar que los componentes de CDD en el Proyecto Educativo del Programa (PEP) de la Licenciatura en Educación Infantil (Universidad de La Guajira, 2016), fueron examinados de acuerdo con las tres dimensiones de análisis de la gestión curricular propuestas por López (2010), a saber: Integralidad, Flexibilidad, e Interdisciplinariedad.

Dimensión 1. Integralidad del currículo: Esta dimensión aporta al desarrollo de la CDD en la medida en que comprende el reto de crear espacios de formación que le permitan a los futuros docentes afrontar integralmente los problemas del entorno educativo, tal como lo plantea López (2010) quien define la integralidad en función de la "coherencia, cumplimiento y pertinencia de los contenidos del currículum para la finalidad competencial programada, así como la posibilidad de que con los mismos se pueda dar respuesta a los problemas con que se encontrarán los profesionales a su finalización" (p. 329).

Dimensión 2. Flexibilidad curricular: Esta dimensión corresponde al reto de diseñar mecanismos pedagógicos y procesos de actualización curricular que permitan satisfacer las necesidades y expectativas de los estudiantes, unido al diseño de diferentes rutas curriculares adaptadas para el desarrollo de la CDD, incrementando a su vez el nivel de estandarización de los procesos educativos y el mejoramiento de la calidad (Rohlehr, 2006; López, 2010).

Dimensión 3. Interdisciplinariedad del currículo: Esta dimensión hace referencia a los espacios y condiciones curriculares y extracurriculares, que permiten al estudiante ampliar su mirada multidimensional del contexto educativo, gracias al aporte de las diferentes asignaturas del currículo, el desarrollo de proyectos de aula integradores, la generación de procesos participativos e interdisciplinarios en el análisis de casos y el desarrollo de las prácticas profesionales, los cuales hacen parte de las diferentes estrategias de interacción entre los docentes y estudiantes, construyendo saberes compartidos (López, 2010).

El segundo instrumento seleccionado correspondió a la Rúbrica para evaluar la competencia digital del profesor universitario en el contexto latinoamericano (Lázaro, Gisbert y Silva, 2018), aplicado para la valoración de la CDD en la muestra de (n: 153) estudiantes. La rúbrica presentó un nivel de confiabilidad alfa de cronbach de $(\alpha: 926)$, adicionalmente en relación con la validez y consistencia interna las correlaciones establecidas a nivel ítem-escala oscilaron con un rango comprendido entre ( $r: 571 \mathrm{y} r$ : 758).

La rúbrica está conformada por cuatro dimensiones, a saber: 1) Didáctica, curricular y metodológica, 2) Planificación, organización y gestión de espacios recursos tecnológicos digitales, 3) Relacional, ética y seguridad, y 4) Personal y profesional. Los niveles de la escala también fueron ajustados, en la medida que se incluyó un ítem para un nivel inferior a lo establecido conceptualmente en la escala inicial (Principiante, medio, experto y transformador). En este sentido, para calcular los valores medios de la escala, se tuvo en cuenta los siguientes 5 rangos:

- R1: Inferior (1,00 a 1,79) equivale al nivel novato, que no alcanza el nivel principiante.

Gestión curricular y desarrollo de la competencia digital docente en la formación inicial del profesorado. Alba Ruth Pinto Santos y Adolfina Pérez Garcias.

Página 5 de 21 
- R2: Bajo (1,80 a 2,59) equivale a un nivel principiante

- R3: Medio (2,60 a 3,39) corresponde al nivel medio

- R4: Alto $(3,40$ a 4,19$)$ corresponde al nivel experto

- R5: Superior $(4,20$ a 5,00) corresponde al nivel transformador

En la tabla 1, se presentan los indicadores psicométricos estimados a partir de la aplicación del instrumento, Tal como se puede apreciar, a nivel de la consistencia interna los coeficientes de correlación (ítem-escala) son positivos en todos los casos y oscilan con un rango comprendido entre ( $r: .571$ y $r$ : .745); reflejando así el alto nivel de consistencia interna de la rúbrica. Complementariamente en la última columna se puede evidenciar como si se elimina alguno de los ítems, se disminuiría el nivel estimado a nivel general con el Coeficiente Alpha de Cronbach ( $\alpha$ : .926).

Tabla 1.

Análisis psicométrico de consistencia interna de los ítems en la Rúbrica Evaluativa de la CDD

\begin{tabular}{ccc} 
Indicadores de la Rúbrica & Alfa de \\
& $\begin{array}{c}\text { Correlación } \\
\text { elemento-total } \\
\text { corregida }\end{array}$ & $\begin{array}{c}\text { Cronbach si } \\
\text { se elimina } \\
\text { el elemento }\end{array}$ \\
\hline
\end{tabular}

1.1. Planificación y competencia digital

, 598

,923

1.2. Las tecnologías digitales como facilitadoras del

, 685

,921 aprendizaje.

1.3. Tratamiento de la información y creación de

, 758

,917

conocimiento.

1.4. Evaluación, tutoría y seguimiento de los estudiantes.

2.1. Ambientes de aprendizaje.

,722 $\quad, 919$

,668 $\quad$,920

$, 685 \quad, 920$

2.2. Gestión de tecnologías digitales y aplicaciones.

, 745

,918 académica.

3.1. Ética y seguridad.

,586 923

3.2. Comunicación, difusión y transferencia del ,703 ,920

conocimiento.

3.3. Contenidos digitales y comunidad educativa.

,676 $\quad$,921

4.1. Acceso libre a la información, creación y difusión de $\quad$,609 ,922

material didáctico con licencias abiertas.

4.2. Formación Permanente.

,616 922 $\quad$,922

4.3. Comunidades de aprendizaje virtuales: formales, no

, 674

,921

formales e informales.

4.4. Entorno personal de aprendizaje (EPA).

, 571

,923

Fuente: Elaboración propia.

\section{Procedimiento}

Para la implementación del enfoque evaluativo, primero se seleccionaron y diseñaron los instrumentos de recolección de datos, luego se desarrolló el análisis curricular y la valoración de la CDD con los estudiantes. Adicionalmente, se configuró la muestra de estudiantes aplicando los criterios de inclusión definidos previamente.

Gestión curricular y desarrollo de la competencia digital docente en la formación inicial del profesorado. Alba Ruth Pinto Santos y Adolfina Pérez Garcias. 
Posteriormente se adelantó la aplicación de los dos instrumentos (1. Inventario de observación de indicadores de la gestión curricular de la CDD en la FID y 2. Rúbrica para evaluar la competencia digital del profesor universitario en el contexto latinoamericano), previa solicitud del consentimiento informado a cada uno de los participantes.

Finalmente, se diseñó la base de datos en función de las categorías y códigos de respuesta establecidos para cada uno de los ítems al interior de los dos instrumentos aplicados y se adelantó el procesamiento de la información, siguiendo las especificaciones cualitativas del análisis de contenidos desde una perspectiva de investigación evaluativa y con el soporte del programa SPSS para el análisis cuantitativo.

\section{Resultados}

A continuación, se presentan los principales hallazgos derivados del análisis de la gestión curricular y la valoración de la CDD de los estudiantes de la FID. Inicialmente se toma como punto de partida la caracterización y análisis de la gestión curricular de la CDD al proyecto educativo del programa Licenciatura en Educación Infantil. Posteriormente se presenta el análisis correspondiente al diagnóstico de la CDD a partir de la evaluación generada con la rúbrica.

\section{Caracterización curricular del programa de Licenciatura en Educación Infantil}

En primera instancia, se presenta la caracterización de las principales tendencias identificadas en la gestión curricular del programa de Licenciatura en Educación Infantil, profundizando en las tres dimensiones consideradas (integralidad, flexibilidad e interdisciplinariedad).

En la tabla 2, se evidencia que dentro de la dimensión Integralidad del Currículo se ha contemplado para la formación del futuro docente las competencias asociadas con el uso adecuado de la tecnología digital en el ejercicio profesional docente. No obstante, la formación en CDD se reduce a dos cursos de formación, y una electiva sobre herramientas digitales. En relación a la dimensión Flexibilidad del currículo, falta claridad en la planeación docente frente a los procesos transversales para la formación en apropiación y uso de las TIC con sentido pedagógico, y no se observan procesos de formación y evaluación de la CDD de manera flexible y situada a la realidad de los estudiantes. Frente a la dimensión Interdisciplinariedad, el currículo contempla la articulación de saberes a través de los diferentes campos de formación y la transversalidad de la tecnología digital, No obstante, la reduce a desarrollar habilidades en el manejo instrumental de las TIC.

Tabla 2.

Análisis de la gestión curricular de la CDD en la FID

\begin{tabular}{|l|l|l|}
\hline Dimensión & Criterio & Observación \\
\hline $\begin{array}{l}\text { Integralidad } \\
\text { currículo }\end{array}$ & $\begin{array}{l}\text { del El proyecto } \\
\text { educativo del programa } \\
\text { considera en los objetivos } \\
\text { el desarrollo de la CDD. }\end{array}$ & $\begin{array}{l}\text { El PEP contempla dentro de las } \\
\text { competencias profesionales del } \\
\text { educador los conocimientos, habilidades } \\
\text { y destrezas en el uso de las TIC, y } \\
\text { establece dentro de los objetivos de la } \\
\text { formación del educador infantil el }\end{array}$ \\
\hline
\end{tabular}

Gestión curricular y desarrollo de la competencia digital docente en la formación inicial del profesorado. Alba Ruth Pinto Santos y Adolfina Pérez Garcias.

Página 7 de 21 


\begin{tabular}{|c|c|c|}
\hline & & $\begin{array}{l}\text { manejo de las TIC y los usos asociados } \\
\text { a procesos de gestión de conocimiento y } \\
\text { actualización permanente. También } \\
\text { enfatiza en implementar la tecnología } \\
\text { digital como medios y mediaciones para } \\
\text { la enseñanza y el aprendizaje de los } \\
\text { contenidos propios de la educación } \\
\text { infantil. }\end{array}$ \\
\hline & $\begin{array}{l}1.2 \text { El plan de estudios de } \\
\text { la carrera incorpora } \\
\text { asignaturas vinculadas a la } \\
\text { CDD. }\end{array}$ & $\begin{array}{l}\text { El plan de estudios incluye dos } \\
\text { asignaturas relacionadas con la CDD, } \\
\text { cada una con } 2 \text { créditos académicos, las } \\
\text { cuales son: TIC, uso, creatividade } \\
\text { innovación, y Redes académicas y } \\
\text { gestión del conocimiento. }\end{array}$ \\
\hline & $\begin{array}{l}\text { 1.3 Las guías docentes de } \\
\text { las asignaturas incluyen } \\
\text { metodologías r que } \\
\text { posibilitan el desarrollo de } \\
\text { la CDD. }\end{array}$ & $\begin{array}{l}\text { En la planeación docente no es evidente } \\
\text { la apropiación pedagógica de las TIC. } \\
\text { Hay algunas iniciativas de docentes en } \\
\text { relación a propuestas de innovación } \\
\text { educativa que no permean el currículo } \\
\text { de la FID. }\end{array}$ \\
\hline & $\begin{array}{l}1.4 \text { En las asignaturas } \\
\text { relacionadas con } \\
\text { tecnología educativa se } \\
\text { promueven actividades y } \\
\text { prácticas asociadas a la } \\
\text { apropiación pedagógica de } \\
\text { la tecnología digital. }\end{array}$ & $\begin{array}{l}\text { Se evidencia en las asignaturas TIC, uso, } \\
\text { creatividad e innovación, y Redes } \\
\text { académicas y gestión del conocimiento } \\
\text { actividades y talleres que potencian la } \\
\text { CDD. }\end{array}$ \\
\hline \multirow[t]{4}{*}{$\begin{array}{l}\text { Flexibilidad del } \\
\text { currículo }\end{array}$} & $\begin{array}{l}2.1 \quad \text { Las diferentes } \\
\text { asignaturas aportan al } \\
\text { estudiante conocimientos } \\
\text { para aprovechar la TIC en } \\
\text { el ejercicio profesional. }\end{array}$ & $\begin{array}{l}\text { Las asignaturas TIC, uso, creatividad e } \\
\text { innovación, y Redes académicas y } \\
\text { gestión del conocimiento desarrollan } \\
\text { ámbitos temáticos que aportan } \\
\text { conocimientos sobre aprovechamiento } \\
\text { de las TIC a nivel profesional. }\end{array}$ \\
\hline & $\begin{array}{l}\text { 2.2 La actualización de los } \\
\text { contenidos del plan de } \\
\text { estudios es permanente y } \\
\text { se articula a las } \\
\text { transformaciones del rol } \\
\text { docente. }\end{array}$ & $\begin{array}{l}\text { La última actualización del plan de } \\
\text { estudios fue realizada en el año 2016, y } \\
\text { la anterior en el año 2012. No se } \\
\text { incluyen actualización que reflejen } \\
\text { ajustes de acuerdo a las necesidades } \\
\text { profesionales actuales. }\end{array}$ \\
\hline & $\begin{array}{l}2.3 \text { El programa aplica } \\
\text { instrumentos para evaluar } \\
\text { los avances en los niveles } \\
\text { de CDD. }\end{array}$ & $\begin{array}{l}\text { No se encontraron antecedentes de } \\
\text { evaluación de la CDD en el programa. }\end{array}$ \\
\hline & $\begin{array}{l}2.4 \text { El programa } \\
\text { cursos electivos } \\
\text { potencian la CDD. }\end{array}$ & $\begin{array}{l}\text { El programa oferta una asignatura } \\
\text { electiva denominada Didáctica de las } \\
\text { TIC que tiene dos créditos académicos. }\end{array}$ \\
\hline Interdisciplinariedad & $\begin{array}{lr}3.1 \text { Los diferentes campos } \\
\text { de formación ofrecen } \\
\text { condiciones } \\
\text { oportunidades para } \quad \text { el } \\
\text { desarrollo de la CDD. }\end{array}$ & $\begin{array}{l}\text { No hay mayor profundidad y } \\
\text { orientaciones sobre cómo desarrollar la } \\
\text { CDD. Aunque el uso de las TIC se } \\
\text { encuentra asociado al eje de } \\
\text { competencias transversales, en la } \\
\text { descripción se reduce a un curso }\end{array}$ \\
\hline
\end{tabular}

Gestión curricular y desarrollo de la competencia digital docente en la formación inicial del profesorado. Alba Ruth Pinto Santos y Adolfina Pérez Garcias. 


\begin{tabular}{|l|l|l|}
\hline & $\begin{array}{l}\text { complementario de Herramientas } \\
\text { Informáticas, que evidencia una } \\
\text { formación instrumental de las TIC que } \\
\text { no comprende la amplitud de la CDD. }\end{array}$ \\
\hline $\begin{array}{l}3.2 \text { Desde los diferentes } \\
\text { campos de formación y } \\
\text { disciplinas se promueve la } \\
\text { interacción de estudiantes } \\
\text { en diferentes plataformas y } \\
\text { ambientes de aprendizaje } \\
\text { que incluyen pedagogías } \\
\text { emergentes. }\end{array}$ & $\begin{array}{l}\text { El Perramientas y recursos educativos } \\
\text { digitales para el desarrollo de los } \\
\text { procesos pedagógicos de acuerdo a la } \\
\text { articulación de los campos de } \\
\text { formación. No obstante, en la } \\
\text { planeación docente no se evidencia de } \\
\text { manera explícita el tipo de recursos ni la } \\
\text { metodología. Tampoco se identifica el } \\
\text { apoyo para garantizar condiciones de } \\
\text { acceso a la tecnología digital a } \\
\text { estudiantes y docentes. }\end{array}$ \\
\hline
\end{tabular}

Fuente: Elaboración propia.

En este sentido se considera que se están cumpliendo las indicaciones frente a los contenidos curriculares establecidos en la resolución 18583 (Ministerio de Educación de Colombia, 2017) que considera que la Instituciones de Educación Superior (IES) deben tener los fundamentos teóricos, pedagógicos y didácticos que permita al egresado la "apropiación y uso pedagógico de mediaciones educativas propias de la educación abierta y a distancia, con el uso de medios masivos de comunicación y tecnologías de la información y la comunicación" (p. 9). No obstante, en las diferentes dimensiones de la gestión curricular, falta mayor articulación sobre los procesos integrales, flexibles e interdisciplinares para avanzar en el desarrollo de la CDD, dado que se presentan solamente dos asignaturas orientadas específicamente al desarrollo de la CDD y otros cursos electivos que tienen una incidencia indirecta porque se orientan puntualmente al uso instrumental de las TIC, unido a las limitaciones identificadas en el desarrollo de las prácticas pedagógicas frente a la apropiación efectiva de las TIC.

\section{Valoración del Nivel de Desarrollo de la CDD}

A continuación, se presenta el análisis descriptivo de la rúbrica de evaluación de la CDD a estudiantes próximos a egresar de la FID. En la tabla 3 se presenta los promedios, desviaciones y total de casos válidos por ítem con una escala de valoración ( 1 a 5 ) donde (1) corresponde al nivel inferior y (5) corresponde al nivel superior.

Tabla 3.

Análisis descriptivo de los indicadores en la Rúbrica Evaluativa de la CDD

\begin{tabular}{lrr}
\multicolumn{1}{c}{ Indicadores de la Rúbrica de Evaluación } & Media & $\begin{array}{c}\text { Desviación } \\
\text { típica }\end{array}$ \\
\hline 1.1. Planificación y competencia digital & 2,71 & 1,356 \\
1.2. Las tecnologías digitales como facilitadoras del aprendizaje. & 2,76 & 1,555 \\
1.3. Tratamiento de la información y creación de conocimiento. & 2,36 & 1,417 \\
1.4. Evaluación, tutoría y seguimiento de los estudiantes. & 2,08 & 1,240 \\
2.1. Ambientes de aprendizaje. & 2,37 & 1,213 \\
2.2. Gestión de tecnologías digitales y aplicaciones. & 2,51 & 1,170 \\
2.3. Espacios con tecnologías digitales de la unidad académica. & 2,42 & 1,207
\end{tabular}

Gestión curricular y desarrollo de la competencia digital docente en la formación inicial del profesorado. Alba Ruth Pinto Santos y Adolfina Pérez Garcias. 
3.1. Ética y seguridad.

3.2. Comunicación, difusión y transferencia del conocimiento.

3.3. Contenidos digitales y comunidad educativa.

4.1. Acceso libre a la información, creación y difusión de material didáctico

4.2. Formación Permanente.

4.3. Comunidades de aprendizaje virtuales: formales, no formales e informales.

D1. Didáctica curricular y metodológica (1 a 5)

D2. Planificación, organización y gestión digital (1 a 5)

D3. Relacional, ética y seguridad (1 a 5)

Fuente: Elaboración propia.

Tal como se aprecia en la tabla 3 , los ítems presentan promedios comprendidos en un rango de valoración entre $(2,03$ y 2,71$)$ los cuales se encuentran en su mayoría por debajo del margen establecido en el nivel del rango teórico intermedio R3: (2,60 a 3,39), dentro de la escala de 1 a 5 que corresponde a su vez al nivel medio en el desarrollo de la CDD. El ítem que reflejó el mejor desempeño promedio fue "1.2. Las tecnologías digitales como facilitadoras del aprendizaje" $(X: 2,76)$, mientras que el ítem que presentó el menor nivel de desempeño promedio fue "4.1. Acceso libre a la información, creación y difusión de material didáctico con licencias abiertas" $(X: 2,03)$. En relación con las desviaciones típicas, se puede identificar que el ítem de mayor heterogeneidad fue "1.2. Las tecnologías digitales como facilitadoras del aprendizaje" (Dt: 1,555), mientras que el ítem más homogéneo en las valoraciones fue "4.4. Entorno personal de aprendizaje (EPA)" (Dt: ,978).

A nivel general, en relación con el análisis descriptivo derivado de las cuatro dimensiones (D1 a D4) y la valoración general de la Rúbrica correspondiente al diagnóstico de la CDD, se observan los niveles promedio y desviaciones para cada una las cuatro dimensiones y la valoración general de la Rúbrica. Específicamente, se puede identificar que el mejor nivel de desempeño en la CDD correspondió a la D1. Didáctica curricular y metodológica $(X: 2,48)$ mientras que el desempeño menor correspondió a la D4. Personal y profesional $(X: 2,12)$. Asimismo, la valoración general del diagnóstico DCDD presentó un promedio de $(X: 2,29)$, siendo en todos los casos valores que pertenecen al Rango de Clasificación (R2: Bajo). En todas las dimensiones se encontraron valoraciones comprendidas en el rango original de la escala (1 a 5).

Estas tendencias de calificación cuantitativa se presentan a continuación, con el diagrama de intervalos de confianza para las cuatro dimensiones y el diagnóstico general de evaluación de la CDD, los cuales aportan al análisis gráfico y permiten comparar los rangos centrales de variabilidad entre las cuatro dimensiones y la escala general, evidenciando que las dimensiones 3 (X: 2,13) y 4 (X: 2,15) se encuentran en niveles inferiores al ser comparadas con las dimensiones $1(\mathrm{X}: 2,48)$ y $2(\mathrm{X}: 2,44)$. tal como se ilustran en la figura 2. 


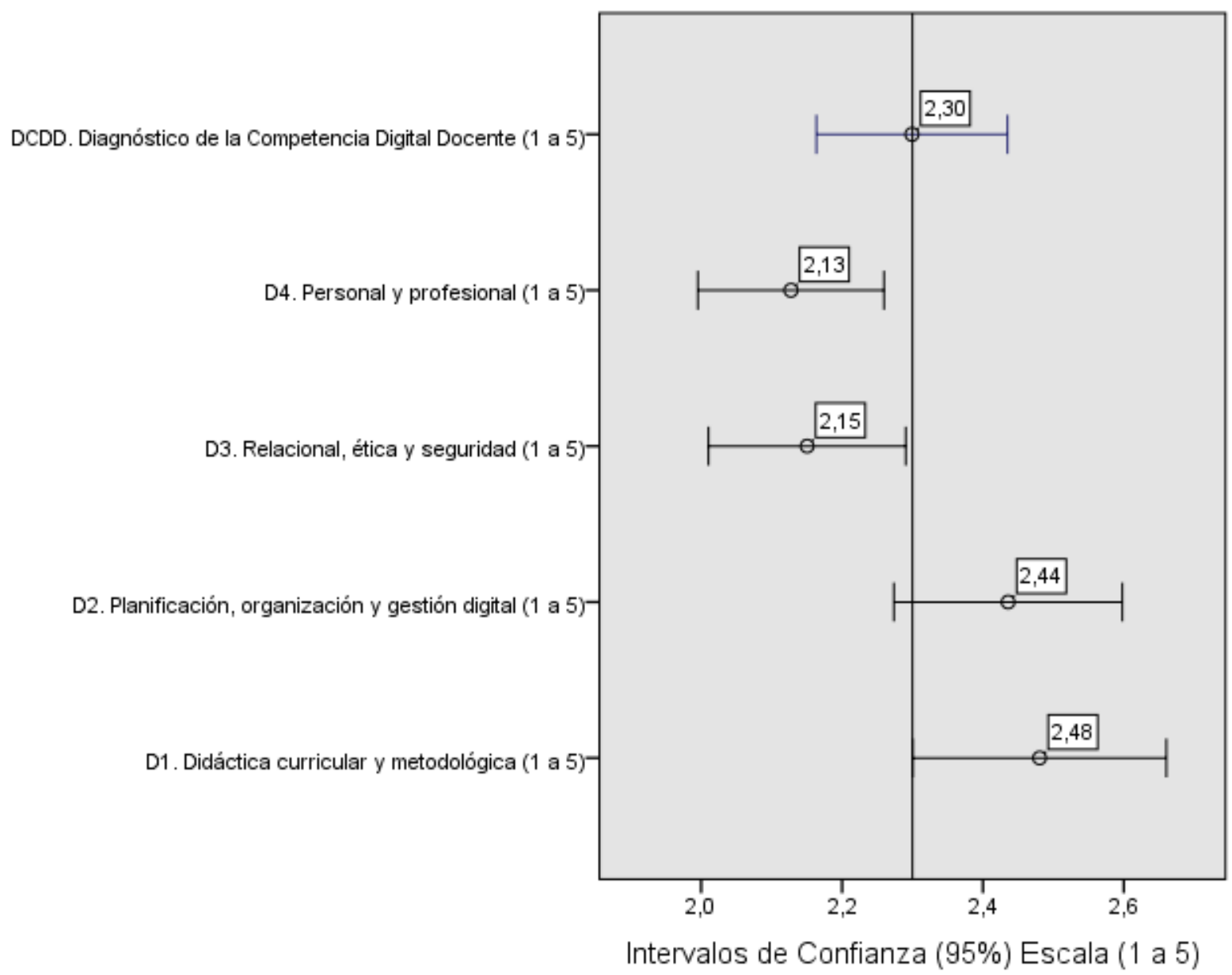

Figura 2. Análisis comparativo de los intervalos de confianza en la evaluación de la CDD Fuente: Elaboración propia.

Complementariamente, se presenta a continuación en la tabla 4, el análisis correlacional bivariado entre las cuatro dimensiones y a su vez las correlaciones entre cada dimensión y el diagnóstico general, este análisis correlacional permite profundizar en la dinámica de relaciones que se pueden establecer frente a las interacciones entre las dimensiones de la CDD. En todos los casos se evidencia que las correlaciones estimadas fueron directamente proporcionales y estadísticamente significativas al 99\% de confianza. Para los casos correspondientes a las correlaciones estimadas entre las cuatro dimensiones el rango oscila entre ( $r: .627$ y $r: .812$ ), mientras que el rango de correlaciones estimadas entre cada dimensión y la valoración del diagnóstico general oscila entre ( $r: .833 \mathrm{y} r$ : .925). Se presentan las correlaciones bivariadas entre las cuatro dimensiones y la valoración del diagnóstico general de la CDD. En todos los casos, las correlaciones son directamente proporcionales y estadísticamente significativas al $99 \%$ de confianza. 
Tabla 4.

Análisis correlacional bivariado entre las cuatro dimensiones y el diagnóstico de la CDD

\begin{tabular}{|c|c|c|c|c|c|}
\hline & & $\begin{array}{c}\text { D1. } \\
\text { Didáctica } \\
\text { curricular y } \\
\text { metodológica } \\
\text { (1 a 5) } \\
\end{array}$ & $\begin{array}{c}\text { D2. } \\
\text { Planificación, } \\
\text { organización } \\
\text { y gestión } \\
\text { digital (1 a 5) } \\
\end{array}$ & $\begin{array}{c}\text { D3. } \\
\text { Relacional, } \\
\text { ética y } \\
\text { seguridad } \\
(1 \text { a 5) } \\
\end{array}$ & $\begin{array}{c}\text { D4. } \\
\text { Personal y } \\
\text { profesional } \\
(1 \text { a } 5) \\
\end{array}$ \\
\hline \multirow{4}{*}{$\begin{array}{c}\text { D2. } \\
\text { Planificación, } \\
\text { organización y } \\
\text { gestión digital } \\
\text { (1 a 5) }\end{array}$} & Correlación de &, $812^{* * *}$ & & & \\
\hline & Pearson & & & & \\
\hline & Sig. (bilateral) & ,000 & & & \\
\hline & $\mathrm{N}$ & 153 & & & \\
\hline \multirow{3}{*}{$\begin{array}{l}\text { D3. Relacional, } \\
\text { ética y seguridad } \\
\text { (1 a } 5)\end{array}$} & $\begin{array}{l}\text { Correlación de } \\
\text { Pearson }\end{array}$ &, $669^{* * *}$ &, $627^{* * *}$ & & \\
\hline & Sig. (bilateral) & ,000 & ,000 & & \\
\hline & $\mathrm{N}$ & 153 & 153 & & \\
\hline \multirow{3}{*}{$\begin{array}{l}\text { D4. Personal y } \\
\text { profesional } \\
\text { (1 a 5) }\end{array}$} & $\begin{array}{l}\text { Correlación de } \\
\text { Pearson }\end{array}$ &, $684^{* *}$ &, $651^{* *}$ &, $711^{* * *}$ & \\
\hline & Sig. (bilateral) & ,000 & ,000 & ,000 & \\
\hline & $\mathrm{N}$ & 153 & 153 & 153 & \\
\hline \multirow{3}{*}{$\begin{array}{c}\text { DCDD. } \\
\text { Diagnóstico de } \\
\text { la Competencia } \\
\text { Digital Docente } \\
\text { (1 a 5) }\end{array}$} & $\begin{array}{l}\text { Correlación de } \\
\text { Pearson }\end{array}$ & ,925 &, $883^{* *}$ &, $833^{* *}$ &, $861^{* * *}$ \\
\hline & Sig. (bilateral) & ,000 & ,000 & ,000 & ,000 \\
\hline & $\mathrm{N}$ & 153 & 153 & 153 & 153 \\
\hline
\end{tabular}

Fuente: Elaboración propia.

Cabe anotar que se adelantó un análisis correlacional complementario entre el semestre y los rangos del nivel de desarrollo de la CDD, encontrando en todos los casos correlaciones positivas y directamente proporcionales, pero con magnitudes bajas y por lo tanto no alcanzaron a ser estadísticamente significativas. En la figura 3, se ilustra el análisis comparativo de las dimensiones diagnósticas de la CDD en función del semestre. Tal como se puede identificar los niveles más altos de CDD se encuentran en el último semestre (X) con excepción de la (D1). Asimismo, este último semestre obtuvo la mayor valoración en CDD en el promedio de la escala $(1$ a 5$)$ de $(2,47)$ que equivale al rango teórico Bajo, lo cual permite identificar que aunque existe una leve diferencia entre los niveles alcanzados en comparación con los demás semestres, todos los niveles estudiados se encuentran en los rangos Inferior y Bajo. 


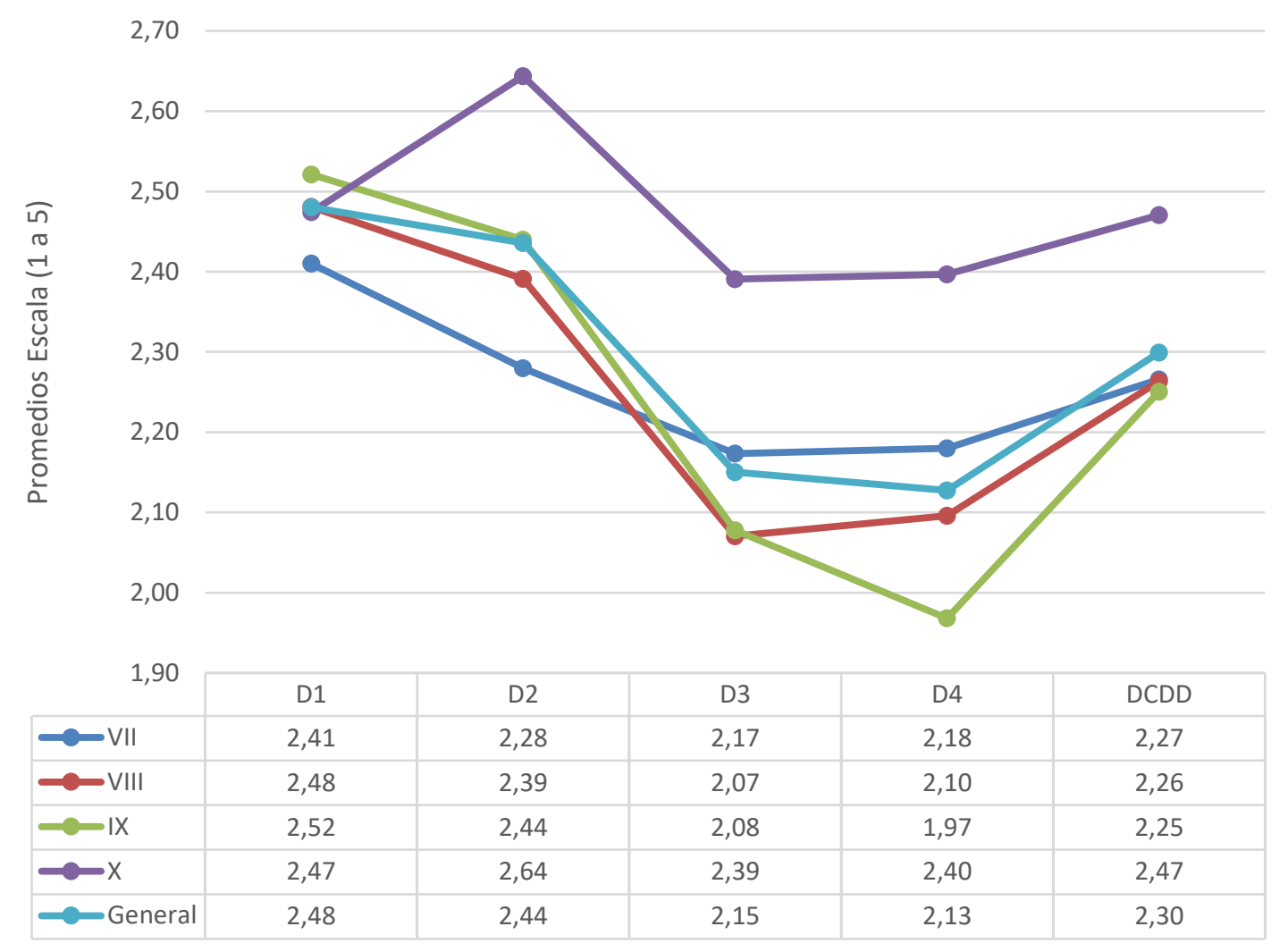

Figura 3. Análisis de las dimensiones diagnósticas de la CDD en función del semestre Fuente: Elaboración propia.

Adicionalmente, en la tabla 5, se presenta un análisis comparativo del diagnóstico sobre la CDD en función del acceso a computador y smartphone. En este caso, se evidencia una tendencia donde las estudiantes que si tienen computador o portátil y smartphone con acceso a internet en casa tienden a presentar mejores promedios en comparación con las estudiantes que no tienen acceso a la conectividad en casa. Los principales contrastes se presentan en la D1. Didáctica curricular y metodológica, y en la D2. Planificación, organización y gestión digital. La única comparación en la cual la tendencia se invierte entre las opciones (SI vs. NO) correspondió a la posibilidad de conectividad con smartphone y la valoración de la D3. Relacional, ética y seguridad. 
Tabla 5.

Análisis comparativo de la CDD en función del acceso a computador y smartphone

Dimensión del Diagnóstico de la

Rúbrica Evaluativa para la CDD
¿Tiene

computador o

portátil en casa

con acceso a

internet?

SI $(X: 2,53)$

¿Tiene teléfono

celular

(smartphone) con acceso a Internet?

D1. Didáctica curricular y metodológica (1 a

NO (X: 2,43)

SI (X: 2,49)

5)

D2. Planificación, organización y gestión

digital (1 a 5)

SI (X: 2,54)

NO (X: 2,43)

D3. Relacional, ética y seguridad (1 a 5)

NO (X: 2,34)

SI (X: 2,48)

SI $(X: 2,14)$

NO (X: 2,22)

NO (X: 2,15)

SI (X: 2,14)

SI (X: 2,18)

NO (X: 2,19)

D4. Personal y profesional (1 a 5)

NO (X: 2,07)

SI (X: 2,14)

SI (X: 2,35)

NO $(\mathrm{X}: 2,03)$

DCDD. Diagnóstico Competencia Digital

NO (X: 2,25)

SI $(\mathrm{X}: 2,31)$

Docente (1 a 5)

NO (X: 2,22)

Fuente: Elaboración propia.

Finalmente, en la tabla 6 , se presentan los resultados correspondientes a la clasificación de los rangos de evaluación diagnóstica de la CDD, tomando como referencia los cinco niveles formulados en el apartado de los instrumentos dentro de las especificaciones metodológicas. Esta tabla permite generar una síntesis global del diagnóstico derivado de la aplicación de la Rúbrica evaluativa para la CDD en las estudiantes que se encuentran adelantando sus prácticas pedagógicas dentro del Programa de Educación Infantil en la Universidad de La Guajira.

Tabla 6.

Análisis de los Rangos Diagnósticos de la CDD derivados de la Rúbrica Evaluativa

\begin{tabular}{cccccc}
\hline Nivel & $\begin{array}{c}\text { D1. Didáctica } \\
\text { curricular y } \\
\text { metodológica }\end{array}$ & $\begin{array}{c}\text { D2. } \\
\text { Planificación, } \\
\text { organización } \\
\text { y gestión } \\
\text { digital }\end{array}$ & $\begin{array}{c}\text { Delacional, } \\
\text { ética y } \\
\text { seguridad }\end{array}$ & $\begin{array}{c}\text { D4. } \\
\text { Personal y } \\
\text { profesional }\end{array}$ & $\begin{array}{c}\text { DCDD. } \\
\text { Diagnóstico } \\
\text { de la } \\
\text { Competencia } \\
\text { Digital } \\
\text { Docente }\end{array}$ \\
\hline Inferior & $33,9 \%$ & $32,0 \%$ & $35,29 \%$ & $37,91 \%$ & $30,7 \%$ \\
\hline Bajo & $25,6 \%$ & $23,5 \%$ & $41,83 \%$ & $38,56 \%$ & $37,3 \%$ \\
\hline Medio & $15,6 \%$ & $28,1 \%$ & $12,42 \%$ & $16,34 \%$ & $19,0 \%$ \\
\hline Alto & $14,4 \%$ & $9,8 \%$ & $5,23 \%$ & $5,88 \%$ & $11,1 \%$ \\
\hline Superior & $10,5 \%$ & $6,5 \%$ & $5,23 \%$ & $1,31 \%$ & $2,0 \%$ \\
\hline Total & $100 \%$ & $100 \%$ & $100 \%$ & $100 \%$ & $100 \%$ \\
\hline
\end{tabular}

Fuente: Elaboración propia.

En términos generales como resultado global del diagnóstico de la CDD se puede apreciar que el 37,3\% de las estudiantes han sido clasificadas en el nivel Inferior, seguidas por un $30,7 \%$ en el primer nivel denominado Bajo. Al interior de cada una de las dimensiones las tendencias centrales fueron las siguientes: D1. Didáctica curricular y metodológica (Nivel Inferior 33,9\%), D2. Planificación, organización y gestión digital (Nivel Inferior

Gestión curricular y desarrollo de la competencia digital docente en la formación inicial del profesorado. Alba Ruth Pinto Santos y Adolfina Pérez Garcias. 
$32 \%$ ), D3. Relacional, ética y seguridad (Nivel Bajo 41,83\%) y D4. Personal y profesional (Nivel Bajo 38,56\%).

\section{Discusión y conclusiones}

En el presente apartado se profundiza de forma analítica y prospectiva en la valoración de los aportes, alcances y limitaciones de la gestión curricular de la formación inicial docente (FID) y la autopercepción del desarrollo de la competencia digital docente (CDD). La perspectiva metodológica del enfoque evaluativo ha generado una serie de hallazgos relevantes, que permiten formular reflexiones y recomendaciones orientadas al mejoramiento continuo de los procesos curriculares, el fortalecimiento de la formación avanzada y especializada para el desarrollo permanente de la CDD, la incorporación de procesos de innovación educativa tanto en el programa como en el desarrollo de las prácticas pedagógicas, contribuyendo así al aseguramiento de la calidad educativa.

Este estudio al analizar los aspectos de la gestión curricular de la FID y su incidencia en el desarrollo de la CDD, hace una nueva contribución en la comprensión de los procesos de la formación de los futuros docentes. En este sentido se encontró que el programa Licenciatura en Educación Infantil contempla en el PEP el desarrollo de la CDD, estableciendo las competencias que debe alcanzar el futuro docente frente a la apropiación de la tecnología educativa y su desarrollo transversal. No obstante, reduce la formación en CDD a dos asignaturas, un curso electivo, y lo flexible e interdisciplinar a simplemente evidenciar competencias en el manejo de herramientas informáticas. Asimismo, en el currículo del programa no está claro cómo garantizar las condiciones y oportunidades para el desarrollo de la CDD, tal como se evidencia en que el 30,7\% de los estudiantes no alcanzan el nivel principiante requerido en la FID.

Se concuerda con Tejada y Pozos (2018) en que es necesario una continuidad ininterrumpida en el desarrollo de la CDD que va desde la formación inicial, la introducción a la profesión, y la formación continua. Asimismo, el futuro docente debe alcanzar las capacidades básicas para usar la tecnología de manera crítica, para el servicio profesional y como apoyo para transformar las prácticas educativas (Cortes, Pinto y Atrio, 2015; Cela-Ranilla, Esteve, Mon, González y Gisbert-Cervera, 2017). También, se considera que es fundamental que los programas formadores de docentes reconozcan la importancia de la CDD en la profesionalización docente y logren atender de mejor manera la FID (INTEF, 2017).

Este estudio encontró que existen barreras para el acceso a dispositivos e internet de buena calidad, ya que solamente el $45 \%$ de los estudiantes tiene computador o portátil en casa con acceso a Internet, y únicamente el $43,1 \%$ tienen acceso a internet de manera permanente. Asimismo, no se contempla a nivel de la gestión curricular la forma de garantizar el acceso y uso a los recursos digitales. Lo anterior evidencia que desde el currículo no se ha pensado la forma en todos los estudiantes tengan las mismas posibilidades de explorar el uso educativo de las TIC y aprovechar la tecnología digital en sus procesos formativos. Estos factores, afectan las actitudes hacia la tecnología educativa, dado que ayuda a que los educadores en formación desarrollen la CDD el ser consumidores, curadores y creadores de contenido digital (O'Byrne \& Hunter-Doniger, 2021; George y Avello-Martínez,2021).

Gestión curricular y desarrollo de la competencia digital docente en la formación inicial del profesorado. Alba Ruth Pinto Santos y Adolfina Pérez Garcias.

Página 15 de 21 
Asimismo, frente a los niveles de CDD, este estudio clasificó a los participantes en 5 niveles en las cuatro dimensiones de desempeño de acuerdo a la rúbrica de evaluación (Lázaro et al., 2018). En este sentido, se encontró que el 30,7 \% de los participantes se encuentra en el Nivel Inferior dado que no tienen las competencias mínimas requeridas en la FID. En relación al desempeño por dimensiones, se encontró que las tendencias centrales fueron las siguientes: D1. Didáctica curricular y metodológica (Nivel inferior 33,9\%), D2. Planificación, organización y gestión digital (Nivel inferior 32\%), D3. Relacional, ética y seguridad (Nivel Principiante 41,83\%) y D4. Personal y profesional (Nivel Principiante 38,56\%).

Al respecto, se considera que deben existir mayores oportunidades de desarrollo de competencias en la formación del profesorado en lo relacionado con la apropiación de las TIC de manera eficaz (Baz, Balçıkanlı \& Cephe, 2018), y que el no contar con las habilidades requeridas al profesional de la educación en la era digital puede afectar la futura integración laboral (Peled, 2021) e incide en la disposición para adoptar pedagogías emergentes en escenarios con fuerte presencia de la tecnología digital.

Se resalta que, si bien es cierto, a nivel nacional existe una normativa que promueve que en la FID se avance en el desarrollo de la CDD (Ministerio de Educación de Colombia, 2017), y que en el PEP (Universidad de La Guajira, 2016) también está descrito como una competencia, a nivel de la gestión curricular no hay claridad en la forma de promover el desarrollo de la CDD, lo cual se evidencia en los resultados de la valoración de la CDD en los estudiantes en el que se clasificaron $30,7 \%$ en nivel inferior y $37,3 \%$ en el nivel principiante de la rúbrica de evaluación. Lo anterior indica que faltan mayores esfuerzos para avanzar a procesos integrales, flexibles, e interdisciplinares para la apropiación de la tecnología digital y se requieren mayores oportunidades en el desarrollo de la CDD desde la gestión curricular.

Se concuerda con Masoumi (2021) sobre los avances en el desarrollo de la CDD en la FID deben ir más allá de un conjunto de conocimientos sobre el uso de determinados programas o artefactos tecnológicos, y que se necesita mayor apoyo para el desarrollo de prácticas pedagógicas que apropien las TIC. Al respecto, los docentes formadores de los programas de la FID y los docentes cooperantes de las prácticas pedagógicas deben ser los principales promotores de la CDD (Admiraal et al., 2016), ya que los futuros docentes necesitan conocimiento del contexto escolar y oportunidades para apropiar procesos de innovación educativa (Guo et al., 2020).

Asimismo, se resalta que la calidad de los procesos de formación en CDD en la FID inciden en las prácticas profesionales de los futuros docentes (Admiraal et al., 2016; Vaillant, 2019; Guo et al., 2020), y el rol de las instituciones educativas formadoras de maestros debe ir más allá de promover conocimientos disciplinares (Ministerio de Educación de Colombia, 2013; Borthwick \& Hansen, 2017). No se trata de realizar cursos independientes sobre tecnología educativa que no se relacionen con las demás asignaturas del currículo (Borthwick \& Hansen, 2017), se pretende un aprovechamiento eficaz de las TIC en la formación profesional del maestro (Graziano et al., 2017; Baz et al., 2018; Sun, Strobel \& Newby, 2017), y reconocer que en los currículos de la FID debe existir contenidos específicos que preparen a los futuros docentes para la realidad del

Gestión curricular y desarrollo de la competencia digital docente en la formación inicial del profesorado. Alba Ruth Pinto Santos y Adolfina Pérez Garcias. 
escenario laboral, desde el contexto y a partir del diálogo reflexivo con comunidades (Rennie \& Anderson, 2019).

Este análisis, permite comprender los retos que presenta la CDD en la FID, las implicaciones que tiene el rol profesional docente ante las necesidades actuales, y la necesidad de transformación del currículo en los programas de formación docente. Es decir, se requiere entender la razón de la lógica del desarrollo de la CDD en la formación profesional docente, no simplemente como un valor agregado, sino como parte de integral de la resignificación del rol docente.

En este sentido, es necesario que se apropie la tecnología educativa en la FID en la gestión curricular, en la medida que tenga más presencia integral, flexible e interdisciplinaria en los programas de la FID, que se pueda experimentar en los escenarios de práctica, y que se tengan referentes de maestros que incorporan las TIC para apoyar procesos de innovación educativa. Asimismo, para realizar propuestas de formación para desarrollar la CDD es necesario analizar las implicaciones de la misma en el perfil profesional del docente, los marcos y estándares nacionales e internacionales, los instrumentos de evaluación y las estrategias integrales que deben ofrecer las instituciones formadoras de maestros, para que estas acciones no queden en la enseñanza instrumental de herramientas, y se centren en potenciar las diferentes dimensiones de la CDD.

A partir de los hallazgos identificados con el proceso de evaluación de la incidencia que tiene la gestión curricular de la FID en la CDD, surgen una serie de interrogantes que pueden ser objeto de investigación y reflexión:

a) ¿Cuál es el nivel real de innovación educativa que ofrecen los currículos universitarios en las Facultades de Educación, para responder a las necesidades profesionales del docente de la era digital?

b) ¿Cuáles son los principales referentes de innovación tecnológica y pedagógica que tienen los futuros docentes, para fortalecer el desarrollo de buenas prácticas educativas en contextos mediados por TIC?

c) ¿Cómo formular, diseñar, implementar y evaluar propuestas integrales de formación en CDD en los futuros docentes?

d) ¿Cuáles serían las mejores estrategias para que docentes en formación, tengan un proceso real y vivencial de experimentación en los escenarios de práctica, para apropiar la tecnología digital a nivel profesional desde una visión activa, crítica y constructiva?

e) ¿Cuál es el estado de capacidad instalada en relación con los recursos digitales que tienen las Instituciones de Educación Superior para facilitar el acceso a estudiantes que no dispongan en su contexto de herramientas TIC?

f) ¿Cómo afecta la brecha digital el desarrollo de la CDD en los futuros docentes?

Presentación del artículo: 28 de septiembre de 2021

Fecha de aprobación: 14 de enero de 2022

Fecha de publicación: 30 de enero de 2022

Gestión curricular y desarrollo de la competencia digital docente en la formación inicial del profesorado. Alba Ruth Pinto Santos y Adolfina Pérez Garcias. 
Pinto-Santos, A. R. y Pérez-Garcias, A. (2022). Gestión curricular y desarrollo de la competencia digital docente en la formación inicial del profesorado. RED. Revista Educación a Distancia, 22(69). http://dx.doi.org/10.6018/red.483551

\section{Financiación}

Este trabajo se ha realizado dentro del proyecto de investigación "Diseño de un modelo formativo para el desarrollo de la competencia digital docente en futuros maestros de La Universidad de La Guajira", el cual hace parte de la tesis doctoral en Tecnología Educativa de Alba Ruth Pinto Santos en la Universidad de Las Islas Baleares. Este estudio ha sido financiado por la Dirección de Investigación de la Universidad de la Guajira, a través de la convocatoria interna resolución No. 0008 del 17 enero de 2018. El código del proyecto es PIUG-2018-038 y el valor financiado fue de 25.000.000 pesos colombianos.

\section{Referencias}

Admiraal, W., van Vuget, F., Kranenburg, F., Koster, B., Smit, B., Weijers, S., et al. (2016). Preparing pre-service teachers to integrate technology into K-12 instruction: Evaluation of a technology-infused approach. Technology, Pedagogy and Education, 26(1), 105-120. https://doi.org/10.1080/1475939X.2016.1163283

Amaya, A., Zúñiga, E., Salazar, M., \& Ávila, A. (2018). Empoderar a los profesores en su quehacer académico a través de certificaciones internacionales en competencias digitales. Apertura, 1, 104-115. https://doi.org/10.32870/ap.v10n1.1174.

Baz, E., Balçıkanlı, C., \& Cephe, P. (2018). Introducing an innovative technology integration model: Echos from ELF pre-service teachers. Education and Information Technologies, 23, 2179-2200. https://doi.org/10.1007/s10639-018-9711-9

Borthwick, A., \& Hansen, R. (2017). Digital literacy in teacher education. Are teacher educators competent? Journal of Digital Learning in Teacher Education, 33(2), 4648. https://doi.org/10.1080/21532974.2017.1291249

Buss, R, Wetzel, K., Foulger, T y Lindsey, L. (2015). Preparing Teachers to Integrate Technology Into K-12 Instruction: Comparing a Stand-Alone Technology Course With a Technology-Infused Approach. Journal of Digital Learning in Teacher Education, 31 (4), 160-172. https://doi.org/10.1080/21532974.2015.1055012

Cabero-Almenara, J. y Martínez, A. (2019). Las Tecnologías de la Información y Comunicación y la formación inicial de los docentes. Modelos y competencias digitales. Profesorado. Profesorado, 23(3), 247-268. https://doi.org/10.30827/profesorado.v23i3.9421

Castañeda, L., Esteve, F., \& Adell, J. (2018). ¿Por qué es necesario repensar la competencia docente para el mundo digital?. Revista De Educación a Distancia, 18(56). http://dx.doi.org/10.6018/red/56/6

Cela-Ranilla, J., Esteve, V., Mon, E., González, J. \& Gisbert-Cervera, M. (2017). El docente en la sociedad digital: una propuesta basada en la pedagogía transformativa y en la tecnología avanzada. Profesorado. Revista de Currículum y Formación de

Gestión curricular y desarrollo de la competencia digital docente en la formación inicial del profesorado. Alba Ruth Pinto Santos y Adolfina Pérez Garcias. 
Profesorado, $21 \quad$ (1), 403-422. Disponible en: https://www.redalyc.org/pdf/567/56750681020.pdf

Cortes, O., Pinto. A, \& Atrio, S. (2015). E-portfolios as a tool for active constructionist learning in educational technology. Lasallista of Research Journal, 12(2), 36-44. https://doi.org/10.22507/rli.v12n2a4

Escudero, T. (2009). Some relevant topics in educational evaluation research. En Mathematical Physics and Field Theory. Julio Abad, in Memoriam, 223- 230, España, Prensas Universitarias de Zaragoza.

Escudero, T. (2016). La investigación evaluativa en el Siglo XXI: Un instrumento para el desarrollo educativo y social cada vez más relevante. RELIEVE. Revista Electrónica de Investigación y Evaluación Educativa, 22(1), 1-21. https://www.redalyc.org/pdf/916/91649056015.pdf

Esteve, F., Castañeda, L., \& Adell, J. (2018). Un Modelo Holístico de Competencia Docente para el Mundo Digital. Profesorado, 32. 105-116. http://repositori.uji.es/xmlui/handle/10234/174771

Ferreiro, A. A. (2018). Aprendizaje basado en proyectos para el desarrollo de la competencia digital docente en la formación inicial del profesorado. Relatec, 17(1), 9-24. http://hdl.handle.net/10662/7994

Flores Lueg, C. (2014). Competencia digital docente: desempeños didácticos en la formación inicial del profesorado. Hachetetepé, 9 , $55-70$ https://doi.org/10.25267/Hachetetepe.2014.v2.i9.6

INTEF. (2017). Common Framework of Digital Teaching Competence. https://aprende.intef.es/sites/default/files/2018-05/2017_1024-Common-DigitalCompetence-Framework-For-Teachers.pdf

Kaplon-Schilis, A., Lyublinskaya, I. (2020). Analysis of Relationship Between Five Domains of TPACK Framework: TK, PK, CK Math, CK Science, and TPACK of Pre-service Special Education Teachers. Tech Know Learn 25, 25-43. https://doi.org/10.1007/s10758-019-09404-X

George, C. E., y Avello-Martínez, R. (2021). Alfabetización digital en la educación. Revisión sistemática de la producción científica en SCOPUS. RED. Revista Educación a Distancia, 21(66). http://dx.doi.org/10.6018/red.444751

Guo L., Ellis M. \& Hong H. (2020) Preparando a los maestros en formación para integrar la tecnología en las aulas de idiomas. En: Tan S., Chen SH. (eds) Transformando la enseñanza y el aprendizaje en la educación superior. Springer, Singapur. https://doi.org/10.1007/978-981-15-4980-9_7

Graziano, K., Herring, M., Carpenter, J., Smaldino, S. \& Finsness, E. (2017). A TPACK diagnostic tool for teacher education leaders. TechTrends, 61, 372-379. https://doi.org/10.1007/s11528-017-0171-7

Lázaro, J. L., Gisbert, M., \& Silva, J. E. (2018). Una rúbrica para evaluar la competencia digital del profesor universitario en el contexto latinoamericano. Edutec, Revista $\begin{array}{lllll}\text { Electrónica de Tecnología } & \text { Educativa, } & 0(63), & \text { 1-14. }\end{array}$ https://doi.org/10.21556/edutec.2018.63.1091 
López, R., N. M. (2010). Gestión curricular por Competencias en Educación Superior: el diagnóstico de factores académicos" DIFA", una propuesta de Autoevaluación Institucional desde el Modelo Socio Formativo: el caso UNIMET. https://eprints.ucm.es/id/eprint/60871/

Luik, P., Taimalu, M. \& Suviste, R. (2018). Perceptions of technological, pedagogical and content knowledge (TPACK) among pre-service teachers in Estonia. Educ Inf Technol 23, 741-755. https://doi.org/10.1007/s10639-017-9633-y

Pinto, A., Pérez, A. y Darder, A. (2020). Revisión sistemática de la literatura sobre competencia digital docente en la formación inicial del profesorado. En E. Colomo, E. Sánchez, P. Ruiz, J. Sanchez. (Eds), La tecnología como eje del cambio metodológico (pp. 513-517). UMA Editorial. https://dialnet.unirioja.es/servlet/articulo?codigo $=7832574$

Pinto, A., Pérez, A. y Darder, A. (2021). Modelo TEP para el desarrollo de la competencia digital docente en la formación inicial docente. En J. Romero, M. Ramos, C. Rodríguez, J. Sola. (Eds), Escenarios educativos investigadores: hacia una educación sostenible (pp.182-189).

https://dialnet.unirioja.es/servlet/articulo?codigo=7898474

Dykinson.

Pozas, M. \& Letzel, V. (2021). “Do You Think You Have What it Takes?” - Exploring Predictors of Pre-Service Teachers' Prospective ICT Use.Tech Know Learn. https://doi.org/10.1007/s10758-021-09551-0

Ramírez-Montoya, M. S., Mena, J., \& Rodríguez-Arroyo, J. A. (2017). In-service teachers' self-perceptions of digital competence and OER use as determined by a xMOOC training course. Computers in Human Behavior, 77, 356-364. https://doi.org/10.1016/j.chb.2017.09.010

Masoumi, D. (2021). Situating ICT in early childhood teacher education. Educ Inf Technol 26, 3009-3026. https://doi.org/10.1007/s10639-020-10399-7

Ministerio de Educación de Colombia (2013). Competencias TIC para el desarrollo profesional docente. https://www.mineducacion.gov.co/1759/articles339097_archivo_pdf_competencias_tic.pdf

Ministerio de Educación de Colombia (2017). Resolución 18583 en el que se establecen las características específicas de calidad de los programas de Licenciatura para la obtención, renovación o modificación del registro calificado. https://normograma.info/men/docs/pdf/resolucion_mineducacion_18583_2017.pdf

O'Byrne, WI., \& Hunter-Doniger, T. (2021). Pre-Service Educators Developing a Digital Identity. TechTrends 65, 444-453. https://doi.org/10.1007/s11528-021-00617-5

Peled, Y. (2021). Autopercepción del profesor en formación sobre la alfabetización digital: el caso de Israel. Educ Inf Technol 26, 2879-2896. https://doi.org/10.1007/s10639-020-10387-x

Rohlehr, B. (2006, del 11 al 13 de mayo). Características del currículo y la gestión curricular: un estudio. Santiago de Chile: Oficina Regional de Educación de la UNESCO para América Latina y el Caribe (Ponencia). Segunda Reunión del Comité Intergubernamental el Proyecto Regional de Educación para América Latina y el Caribe

(PRELAC).

Gestión curricular y desarrollo de la competencia digital docente en la formación inicial del profesorado. Alba Ruth Pinto Santos y Adolfina Pérez Garcias. 
http://www7.uc.cl/sw_educ/educacion/grecia/plano/html/pdfs/linea_investigacion/T emas_Transversales_ITT/ITT_095.pdf

Rennie J. \& Anderson P.J. (2019). Preparing Pre-service Teachers to Teach Literacy in Remote Spaces. In: Rennie J., Harper H. (eds) Literacy Education and Indigenous Australians. Language Policy, vol 19. https://doi.org/10.1007/978981-13-8629-9_9

Roll, M. \& Ifenthaler, D. (2021). Competencias digitales multidisciplinarias de los profesores de formación profesional en formación. Empirical Res Voc Ed Train 13, 7. https://doi.org/10.1186/s40461-021-00112-4

Roulston, S., Cowan, P., Brown, M., Austin, R. \& O’Hara, J. (2019). All aboard or still at check-in? Teacher educators' use of digital technologies: Lessons from a small island. Educ Inf Technol 24, 3785-3802. https://doi.org/10.1007/s10639-019-09951$\mathrm{X}$

Tejada, J., y Pozos, K.V. (2018). Nuevos Escenarios y Competencias Digitales Docentes: Hacia la Profesionalización Docente con TIC. Profesorado, ISSN: 1138-414X, 22(1):25-51. https://recyt.fecyt.es/index.php/profesorado/article/view/63620

Tiede, J. (2020). Media-Related Educational Competencies of German and US Preservice Teachers: A Comparative Analysis of Competency Models, Measurements and Practices of Advancement.MedienPädagogik. ProQuest LLC. https://doi.org/10.21240/mpaed/diss.jt.X

Silva, J., Lázaro, J. L., Miranda, P., y Canales, R. (2018). El desarrollo de la competencia digital docente durante la formación del profesorado. Opción, 34 (86), 423-449. https://digital.fundacionceibal.edu.uy/jspui/handle/123456789/288

Sun, Y., Strobel, J. \& Newby, T.J. (2017). The impact of student teaching experience on pre-service teachers' readiness for technology integration: A mixed methods study with growth curve modeling. Education Tech Research Dev65, 597-629. https://doi.org/10.1007/s11423-016-9486-x

UNESCO (2019). Marco de competencias de los docentes en materia de TIC UNESCO. https://unesdoc.unesco.org/ark:/48223/pf0000371024

Universidad de La Guajira. (2016). Proyecto Educativo del Programa Educación Infantil. https://www.uniguajira.edu.co/licenciatura-educacion-infantil-ficha-tecnica

Voogt, J. \& McKenney, S. (2017). TPACK in teacher education: are we preparing teachers to use technology for early literacy?. Technology, Pedagogy and Education, 26:1, 69-83, https://doi.org/10.1080/1475939X.2016.1174730

Vaillant, D. E. (2019). Formación Inicial del Profesorado de Educación Secundaria en América Latina- Dilemas y Desafíos. Profesorado, Profesorado, 23(3), 35-52. https://doi.org/10.30827/profesorado.v23i3.9516

Gestión curricular y desarrollo de la competencia digital docente en la formación inicial del profesorado. Alba Ruth Pinto Santos y Adolfina Pérez Garcias. 